\title{
The Oral Health Section of the Road to Health Chart (RtHC) - how useful is it?
}

SADJ November 2019, Vol. 74 No. 10 p556 - p560

R Cader ${ }^{1}$, S Naidoo ${ }^{2}$

\section{SUMMARY}

Oral diseases are a major worldwide public health problem. Eighty per cent of caries goes untreated, below the $50 \%$ goal set by the South African National Department of Health. $80 \%$ of the SA population depends on state services for oral healthcare. The American Academy of Pediatrics advises commencement of screening when the first tooth appears, or no later than at 12 months of age. The Road to Health Chart (RtHC) is a convenient home-based method of monitoring and improving child health. How effective is it?

\section{Methodology}

A multistage cluster sampling technique selected seventy-seven health professionals as study participants for a review of their RtHC and for questionnaires and focus group interviews.

\section{Results}

243 oral health pages of RtHC's were reviewed. Only $27 \%$ had completed oral health sections, of which dental professionals had better knowledge.

\section{Conclusion}

Those who work in Baby Well clinics are ill-informed and have a lack of knowledge of the relevance and importance of the oral health section of the RtHC, which is not adequately utilized.

\section{INTRODUCTION}

Among the strategies to improve the survival and development of children, as set out by the World Health Organisation (WHO) and the United Nations Children's

Author affiliations:

1. Rugshana Cader: Dipl. $\mathrm{OH}, \mathrm{BOH}$ (UWC), PGDip (Hpe) (UCT), MSC (Dent), Department of Community Oral Health, Faculty of Dentistry, University of the Western Cape, Tygerberg, South Africa. ORCID Number: 0000-0002-6846-6611

2. Sudeshni Naidoo: BDS(Lon), LDS.RCS (Eng), MDPH (Lon), DDPH. RCS (Eng), MChD (Comm Dent), PhD (US), Dipl. Int Research Ethics (UCT), DSc (UWC), Department of Community Oral Health, Faculty of Dentistry, University of the Western Cape, Tygerberg, South Africa.

ORCID Number: 0000-0001-7424-8082

Corresponding author: Rugshana Cader

Department of Community Oral Health, Faculty of Dentistry, University

of the Western Cape, Tygerberg, South Africa.

Email: rcader@uwc.ac.za

Author contributions:

1. Rugshana Cader: Principal researcher - $60 \%$

2. Sudeshni Naidoo: Co-author \& advisor - $40 \%$

\section{ACRONYMS \\ ECC: $\quad$ Early Childhood Caries \\ MGRS: Multicentre Growth Reference Study \\ NDOH: National Department of Health \\ RtHC: Road to Health Chart \\ UNCF: United Nations Children's Fund \\ WHO: World Health Organisation}

Fund (UNCF), are the monitoring of growth patterns, oral rehydration and the promotion of breastfeeding, an adequate food supply, family planning and female education. ${ }^{1}$ The WHO framed a global strategy on child development, specifically designed for developing countries where it identified best practices for child monitoring in terms of measuring growth, feeding practices, nutrition and immunizations.

The child development-monitoring tool, the Road to Health Chart (RtHC) is a recommended method of growth monitoring. It is intended to assist parents, caregivers and health professionals in determining normal targets and any deviations in the growth of the child.

Accurate record keeping is important for continuity and quality of care. Globally, patient-held maternal and/ or child health records for literate and illiterate patients and healthcare personnel have been used since 1973 to track the health status and document the immunisation status of the patients. These records, though designed to record patient health histories and to facilitate continuity of care among healthcare personnel, also empower patients to track their own health. ${ }^{2}$

In 1993, the World Health Organization (WHO) undertook a comprehensive review of the uses of child monitoring charts. The review concluded that the use and interpretation of the charts were sadly lacking.

In response, the WHO Multicentre Growth Reference Study (MGRS) was implemented between 1997 and 2003 to develop international standards for children below five years of age. In South Africa, the RtHC was revised in line with global standards. Since then the chart has been updated four times, with the last update being in 2017. New WHO growth standards and changes in the immunisation schedule necessitated revision of the current $\mathrm{RtHC}$ while simultaneously providing an opportunity for the addition of other health information, including an oral section. 
All children born in South Africa are issued with a $\mathrm{RtHC}$ document that is to be completed by the health professional at every clinic visit. It records essential information on the growth of the child, the immunization history, vitamin A supplementation, deworming, the presence of deciduous teeth and any illnesses.

An oral health section was included in the booklet in 2011. Among other benefits, health professionals can use anthropometric indicators such as milestone developments and teeth present in the mouth as a guideline when parents are unsure of the age of the child. ${ }^{3}$

The American Academy of Paediatric Dentistry ${ }^{4}$ recognized that maternal prenatal oral health, along with infant oral health, is one of the foundations upon which preventive education and dental care must be built to enhance the opportunity for a child to be free from preventable oral diseases.

The newly revised RtHC was implemented by the Department of Health (DoH) in February 2012, which included an oral health section that was based on global WHO standards. ${ }^{5}$ Unfortunately, there was no training of health and dental professionals regarding the completion of the newly revised chart before its implementation.

According to Cloete $^{5}$ the evaluation of the findings in the RtHC on nutritional practices, breast-feeding practices and bottle-fed practices are poorly monitored and not followed up. Although there is little evidence related to poor nutritional and bottle-feeding practices there is clinical consensus among dental professionals that prolonged breast feeding and certain bottle feeding practices increase the risk of early childhood caries (ECC). ${ }^{6}$

This problem and poor nutritional and feeding practices need to be identified early together with other putative risks and protective factors including sociodemographic influences, environmental (fluoride exposure) and behavioural factors (such as feeding and oral hygiene practices). ${ }^{6}$

Early intervention, health promotion messages and school-based programmes can be with integrated with other services and, together with an evidence-based approach, can be contextualised into a response to local oral health needs to address dental caries prevalence rates in children. ${ }^{7}$

The report on the National Children's Oral Health Survey ${ }^{8}$ found that $40 \%$ of six year olds in South Africa are caries-free. This is below the goal of $50 \%$ set by the National Department of Health. ${ }^{7}$ In the Western Cape $82.3 \%$ of children under age 5 presented with dental caries. ${ }^{8}$ This is a major public health problem, as $80 \%$ of children are reliant on public health service for treatment needs. ${ }^{9}$

This paper presents an evaluation of the oral health component of the $\mathrm{RtHC}$ regarding the capturing and monitoring of the oral health information in the Cape Metropolitan region.

\section{METHODOLOGY}

The oral health sections of $243 \mathrm{RtHC}$ booklets were reviewed. The sample size calculation was based on the assumption that half of the RtHC reviewed in $\mathrm{PHC}$ clinics would have incomplete or inaccurate information.

A multistage cluster sampling technique was used to select the study participants for the review of their $\mathrm{RtHC}$, while the primary health care facilities were randomly selected in the Cape Metropolitan region. Seventy-seven health professionals participated in the survey that elicited their opinions regarding completion and usefulness of the oral health section.

For the three parts of the study, the instruments used to collect the data were (i) a structured checklist to determine how complete were the RtHC's, (ii) a questionnaire survey administered to medical doctors, healthcare workers (including dentists, oral hygienists and dental therapists), and (iii) a focus group discussion with health professionals was implemented to elicit their opinions regarding the usefulness of the oral health section in the RtHC booklet. The focus group discussions with 10 participants was audiotaped and transcribed by the researcher.

The Senate Research Ethics Committee of the University of the Western Cape (ref. 14/7/16) as well as the Health Research Committee of the Western Cape Department of Health approved the study.

Signed informed consent was obtained from the parents and health professionals allowing the researcher access to the RtHC's and providing for the health professional to complete the questionnaire. Anonymity and confidentiality was ensured by the allocation of a code to each participant in the study.

\section{RESULTS}

Two hundred and forty-three RtHC booklets were reviewed. Figure 1 summarises the number of children in a particular age group for each visit and records the extent of completion of the oral health section in their RtHC. The largest group consisted of children aged 12-24 months, the smallest group, children aged 48-60 months.

Just over a quarter $(n=66,27 \%)$ of the oral health sections of the RtHC were filled in. Figure 2 shows the number of complete records $(n=66)$ versus the oral health records which had in previous years been completed $(n=10)$.

Seventy-seven health professionals completed the questionnaire. Oral health professionals had the highest awareness (100\%) of the oral health section, while professional nurses had a 70\% awareness of that section, However, $80 \%$ of certified nurses (5) and both the two involved doctors were not even aware of the oral health section in the RtHC (Figure 3).

As regards utilising and completing the oral health section, the dental professionals were the most confi- 
dent $(75 \%)$ and the least confident were the certified nurses (20\%) (Figure 4).

Dental hygienists and dentists found the oral chart easy to use (63\% and $80 \%$ respectively), while health professionals not related to the dental field (nursing professionals, councillors and those who are involved in community service) did not find the oral healthcharting table so user friendly.

The knowledge of oral health practices among the primary nursing staff and community care workers was poor. While the professional nurses were aware of the oral section in the RtHC, this knowledge was not translated into practice in early identification when there was a problem nor by appropriate referral to a dental professional.

Ten health professionals participated in a focus group discussion designed to determine their views and opinions on the oral health section in the RtHC.

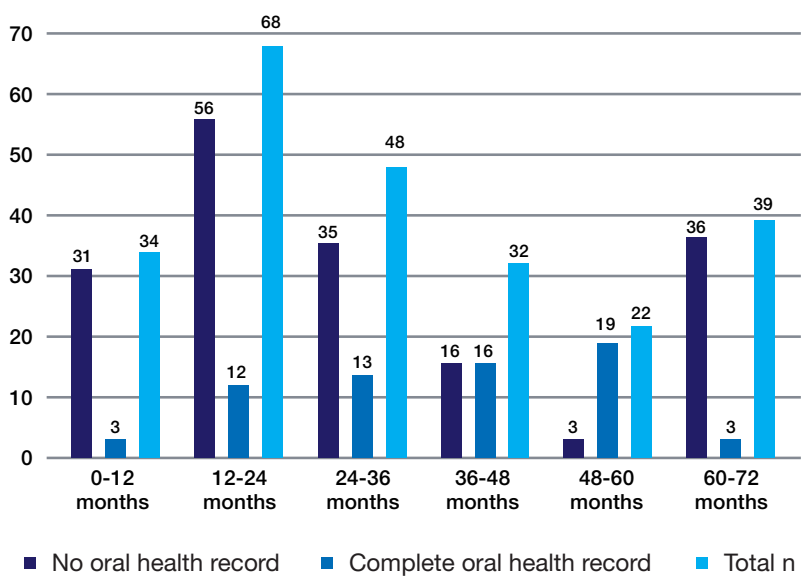

- No oral health record - Complete oral health record - Total $\mathrm{n}$

Figure 1. Completion of Oral health records $(n=243)$.

Health professional

vs.

awareness of oral health section

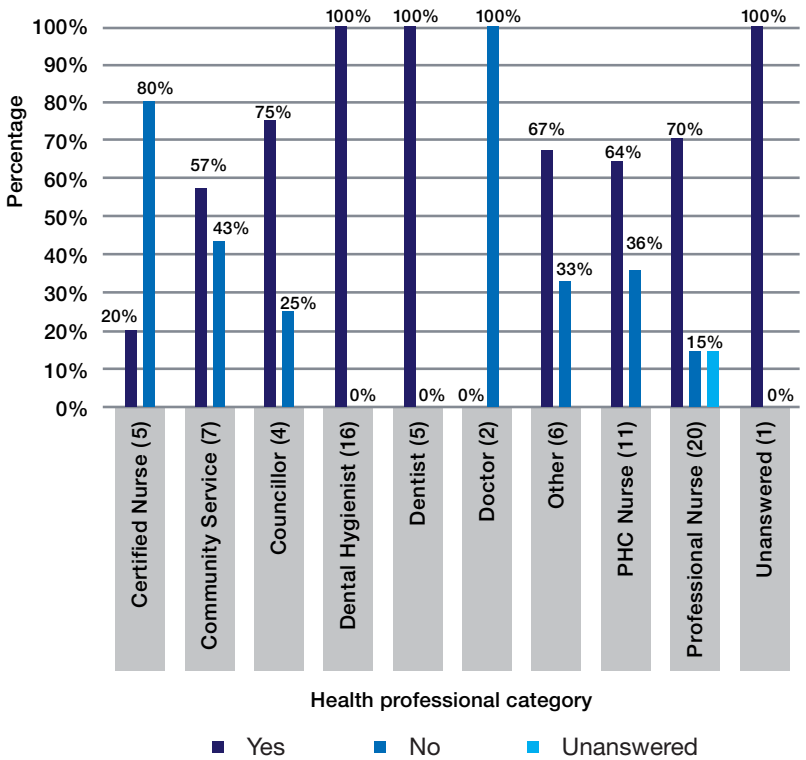

Figure 3. Health professional's awareness of the oral health section, per category.
The focus group was comprised of a doctor, a dentist, four oral hygienists, a dental assistant, a professional nurse, a certified nurse and a community care worker (breastfeeding counsellor). Three broad themes emerged from the discussions - a lack of training and orientation to the RtHC, a lack of structure to the RtHC booklet and a lack of accountability and responsibility amongst the parents.

\section{DISCUSSION}

Monitoring child health is a widely accepted practice and is strongly supported by health professionals, being a standard component of community paediatric services throughout the world. ${ }^{10}$ Its value is twofold - health professionals have a track-record of child development, and secondly, it provides a source of accountability to the carer and a continuity in care. ${ }^{10}$

Healthcare providers at primary health care centres are generally the 'first line' of healthcare workers confronting basic health needs. Parents take their young chil-

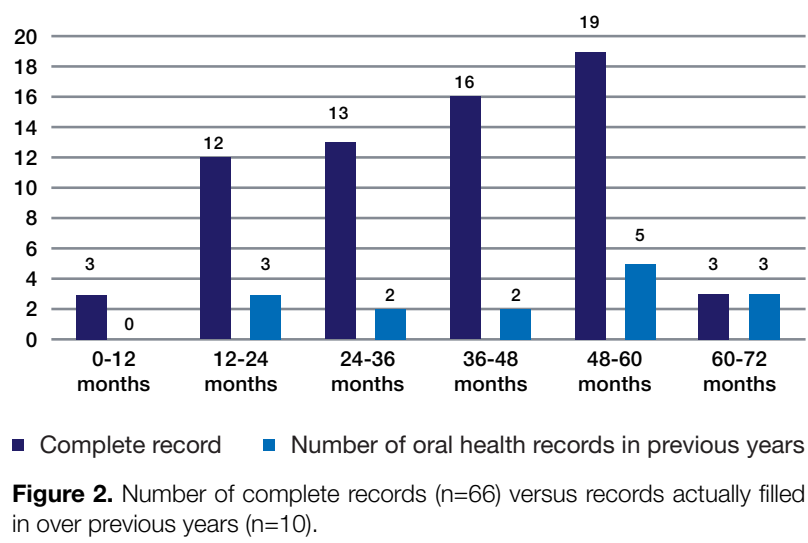

\section{Health professional awareness of oral health section}

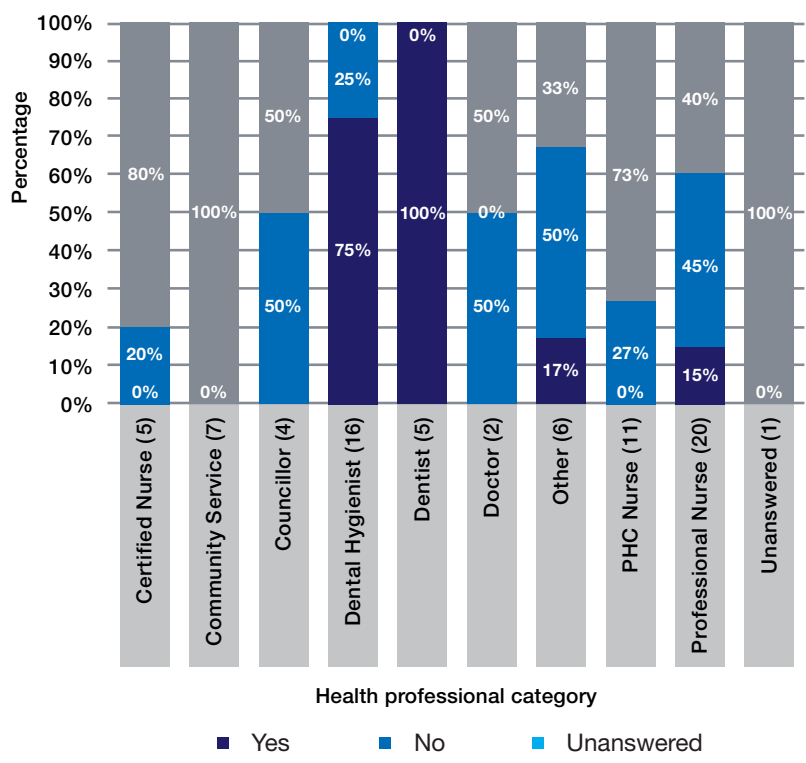

Figure 4. Health professionals' confidence in completing the oral health section of RtHC. 
dren to primary health care clinics for child monitoring, which includes weighing, growth recording, assessment of feeding practices and administering of vaccinations.

Some primary health care facilities have access to a dental professional in their setting, yet the primary health care nurse seldom refers the young children to the dental clinic.

Therefore, an integrated approach to health that includes oral health should be adopted by the public health services and primary health care nurses should be trained to conduct a basic oral examination for early detection of oral diseases as well as other oral-related abnormalities. $^{7}$

\section{Incompleteness of oral health section}

A summary of the completion of the oral health section in the RtHC appears in Figure 1. The data show that just over a quarter (27\%) of the RtHC had the oral health section filled in, while the charts of one hundred and seventy-seven children had not had the oral health section completed at every visit made to the clinic (Figure 1). The largest group consisted of children 12-24 months of age, the smallest of children 48-60 months old.

There was a clear lack of understanding amongst the healthcare workers of the purpose of the inclusion of the oral section in the booklet. The study showed that due to the poor design of the section in the RtHC, dental professionals had to use their own judgement in the completion of this record. This has resulted in inconsistencies, and professionals relying on their own interpretation of the information sought in the oral health section in the RtHC.

The allied health professionals not associated with dentistry indicated it was not their job to complete the oral section. However, other sections such as recording breast-feeding practices, health promotion messages, dietary factors and milestone developments were also often incomplete.

\section{Lack of knowledge of the non-dental participants}

During the focus group discussions, the nursing professionals indicated that a dental professional should complete the oral section. They reported that after training it was their responsibility to coach the rest of the nursing and allied members. However, while training was in breastfeeding, infant guidelines, weighing and immunization, nurses were never informed of nor trained in using the oral health section in the RtHC. Medical doctors took no responsibility for the RtHC other than completing their own notes of child illness and left the entries recording the developmental, health promotion and dietary factors solely to the nurses.

\section{Focus group discussions}

The following health care workers participated in the focus group: a dentist, four oral hygienists, a dental assistant, a professional nurse, a certified nurse, nursing student, community care worker (HIV, TB), and a breastfeeding counsellor.

The majority of health professionals, including dental professionals, indicated they were not orientated, made aware of, nor informed of the use and implementation of the oral health section in the $\mathrm{RtHC}$.

Dental professionals observed that in its present state the oral health section was of no benefit to parents or other health professionals as it lacked clarity and guidance. Nursing professionals reported they were not aware of the oral health section in the RtHC and therefore could not comment on its purpose: "We are yet to see something in writing coming to our clinic informing us of the oral health section of the RtHC".

"If there is no policy, I will not do or implement anything unless I was informed by my superior". This comment is reiterated in a study conducted by Makanyeza et al. ${ }^{11}$ and Balfour ${ }^{12}$ who suggest that success of new policies occurs only when there is consultation with the relevant health professionals on the policy structure and design. The lack of consultation on the structure and implementation of the oral health section in the RtHC has resulted in a lack of accountability among health professionals regarding its use.

The importance of child monitoring as a tool is useful not only for the health professional, but also for parents and carers. However, nurses despaired on the lack of accountability of parents: "The nomadic lifestyles of many of the parents of the children who attend the public health clinics often results in loss or damage to the chart".

"Parents do not look at the chart or show any interest when we write or plot information in the booklet; it's just like a tick list for them." The success of using child-monitoring cards is dependent on parents' knowledge and cooperation, but, if the professionals are unaware of the value and benefits of child monitoring, how will they be able to educate the parents?

\section{CONCLUSIONS}

One of the most important aspects of early childhood development is the quality of life. A child should have access to health care, education, food security, healthy living environment and be pain-free.

If a child's oral health needs are not identified early, discomfort and pain may ensue, impacting on the child's and the parent's qualities of life. The National Department of Health (NDOH) believes that better use of the RtHC would help to move children onto a higher trajectory towards better health and development.

The sampling size was based on the hypothesis that at least half of the RtHC would have been completed, but in the event, the completion rate was significantly low. Of the 243 records that were reviewed, only $2.9 \%$ children had had their mouths examined and the RtHC signed by the time the first tooth had appeared. 
Only $27 \%$ of the two hundred and forty-three RtHC reviewing children from age 0-72 months had the oral section partially completed.

The present study showed a lack of knowledge on the importance of the first oral health visit and of establishing a "dental home" for children. While health professionals were aware of the importance of dental visits for children, they did not see the link between oral health and the importance of good nutrition and feeding practices.

The present study found that there was very poor and inadequate completeness of the oral health section of the RtHC. This outcome was similar to findings by Scherdel et al. ${ }^{13}$, Kitenge ${ }^{3}$ and Mudau ${ }^{1}$ who reported poor completion of child monitoring data.

A solution may be to organise an integrated approach with parents and health professionals, leading to a successful knowledge status, attitude and practices of effective curative, preventive and promotive tools in monitoring child health.

\section{Recommendations}

1. An index page be added to the RtHC directing the health professional and parent to specific information.

2. Oral Health messages be included in the oral section including the importance of the baby teeth, risks of sweet sugar contents in feeding bottles, especially when the child sleeps with the bottle teat in his/ her mouth, graphic images of the dangers of the consumption of sweets, positive oral health messages of good dietary practices, the importance of brushing the teeth of babies with a smear layer of fluoride toothpaste.

3. Protocol or emergency care guidelines covering the situation when a tooth is knocked out of the child's mouth or if a tooth fractures, including referral guidelines for parents and health professionals in the case of other dental emergencies such as dental abscess and toothache.

4. Training for health professionals on the completion of the oral health section of the RtHC, via continuous education programmes.

\section{Limitations}

This was a fairly small sample drawn from only one region in South Africa. A wider investigation could reveal different results.

\section{References}

1. Mudau T, 2010. Utilisation of the Road to Health Chart to improve the health of children under five years of age, s.l.: Pretoria: University of South Africa.

2. Naidoo $H$, Avenant $T$, Goga $A$. Completeness of the Road-to-Health Booklet and Road-to-Health Card: Results of cross-sectional surveillance at a provincial tertiary hospital. S Afr J HIV Med. 2018; 19(1), a765. http://doi.org/10.4102/ sajhivmed.v19i1.765
3. Kitenge GT, 2011. Monitoring of the Road to Health Chart by nurses in the public service at primary healthcare level in the community of Makhado, Limpopo Province South Africa, s.l.: University of Limpopo.

4. American Academy on Pediatric Dentistry Clinical Affairs Committee and American Academy on Pediatric Dentistry, Council on Clinical Affairs, 2014b. Policy on Early Childhood Caries: Classifications, Consequences, and Preventive Strategies, American Academy of Pediatrics, Available from: www. aapd.org/media/policies_guidelines/p_eccclassifications.pdf [accessed 26 June 2015].

5. Cloete I, Daniels L, Jordaan J, Derbyshire C, Volmink L, Schubl C. Knowledge and perceptions of nursing staff on the new Road to Health Booklet growth charts in primary healthcare clinics in the Tygerberg sub-district of the Cape Town metropole district. South African Journal of Clinical Nutrition 2012; 26(3): 141-6.

6. Arora A, Scott JA, Bhole S, Do L, Schwartz E, Blinkhorn AS. Early childhood feeding practices and dental caries in pre-school children. A multi-centre birth cohort study. BMC Public Health 2011, 11: 28 http://www.biomedcentral. com/1471-2458/11/28 Available: [Online accessed 26 June 2015].

7. Singh S. Dental caries rates in South Africa: Implications for oral health planning. Southern African Journal of Epidemiology and Infection 2011; 26(4): 259-61.

8. South African National Oral Health Strategy. www.health. gov.za/index.../130-sd. The Primary Health Care Package. Available [online] 23 June 2015.

9. Smit DA, Osman YI. The availability of the basic oral health care package in the Western Cape. South African Dental Journal 2017, Vol. 72, No. 4 [cited 2018-09-23], 162-6. Available from: <http://www.scielo.org.za/scielo.php?script= sci_arttext\&pid=S0011-85162017000400006\&lng=en\&nrm=iso > . ISSN 0375-1562.

10. Garner P, Panpanich R, Logan S. Is routine growth monitoring effective? A systematic review of trials. Archives of Disease in Childhood 2000; 82(3): 197-201.

11. Makanyeza C, Kwandayi HP, Ikobe B. Strategies to improve service delivery in local authorities. International Journal of Information Technology and Business Management 2013; 15 (1): 1-11

12. Balfour T. 2007. [Online]mhttps://www.dbsa.org/...Municipal\% 20health\%20services\%20in\%20South\%20Africa. Municipal health services in South Africa. Opportunities and challenges. Online: [Accessed: April 2015].

13. Scherdel P, Dunkel L, van Dommelen P, Goulet $O$, Salaün JF, Brauner R, Chalumeau M. Growth monitoring as an early detection tool: a systematic review. The Lancet, Diabetes \& Endocrinology. 2016; 4(5): 447-56. 